I remember when I first got a printer, I spent the best part of a day fiddling with the little DIP-switches inside trying to change the page length. Finally I found a tiny note at the bottom of one page in the middle of a thick manual, which told me that changing the switches had no effect unless you switched the printer off and then on again. I was amazed that anything so completely counter-intuitive would be hidden away like this. Afterwards I tried switching my television off and on when I wanted to change channels, but funnily enough I always got BBC1 ...

In conclusion, I believe that computers have now entered the profesional chess scene permanently, and we will see more computer-based aids in the years to come. ChessBase has carved a niche for itself in this market and I would certainly not be without mine now. But I had better sign off now to do some serious chess work; where's that disc with the $\mathrm{K}+\mathrm{Q} v \mathrm{~K}+\mathrm{R}$ database on it ... ?

\title{
REPORT ON THE 1988 FREDKIN MASTERS OPEN
}

\author{
Murray Campbell, Feng-hsiung Hsu and Gordon Goetsch
}

Carnegie-Mellon University

\section{INTRODUCTION}

At various times in the past decade, the Fredkin Foundation, under the directorship of Dr. Hans Berliner of Carnegie-Mellon University, has sponsored events intended to estimate the strength of the current top chess programs. Along with the support for such tournaments, the Fredkin Foundation is offering a $\$ 10,000$ prize for the first program to produce a consistent 2500 performance, and a $\$ 100,000$ prize for the first program to surpass the human World Champion. The prize of $\$ 5000$ for the first master-level program was awarded to the creators of Belle, Ken Thompson and Joe Condon, in 1983.

The last Fredkin event, the 1985 Fredkin Masters Invitational, had clearly established that Hitech was capable of holding its own against well prepared master-level competition. The 1988 Fredkin Masters Open, held over the weekend of May 28-30, 1988, was organized to pit a number of the current top programs against a similar level of competition. Unfortunately only a few programs were able to obtain the necessary machine time.

\section{THE PROGRAMS}

Four programs participated in the tournament:

- Hitech: Rated 2376 USCF going into this tournament (and having since surpassed 2400); Hitech has the highest established computer rating. Hitech has special purpose hardware that allows search speeds of up to 150,000 nodes/second, and has a number of programmable feature recognizers for evaluating positions. A number of people have worked on Hitech, including Hans Berliner, Carl Ebeling, Gordon Goetsch, Murray Campbell, Larry Slomer, Andy Palay and Andy Gruss. Hitech was developed at Carnegie-Mellon University.

- ChipTest: The current North American computer champion, ChipTest also has special purpose hardware that, running on a Sun 4, can reach about 500,000 nodes/second. ChipTest has a very simple evaluation function, but a rather complex searching algorithm, described in part in Anantharaman et al., (1988). ChipTest was unrated going into the Fredkin tournament. The main authors of ChipTest are Thomas Anantharaman, Feng-hsiung Hsu and Murray Campbell, all at Carnegie-Mellon University. 
- BP: Authored by Robert Cullum of CS DataService, Inc.; BP ran on a Compaq 386 personal computer. BP played in the 1987 North American Computer Chess Championship, where it finished with 1.5 out of 4 games.

- Deep Thought 0.01: Created by the same team as ChipTest, Deep Thought is ChipTest's successor. Deep Thought was not fully debugged for the Fredkin tournament (in fact, the hardware was still being wire-wrapped the day before the tournament), and was running at about half speed (approximately $500,000-700,000$ nodes/second on a Sun 4). Although Deep Thought has a more complex evaluation than ChipTest, it was completely untuned for the Fredkin tournament. One result of this was an unfortunate tendancy to advance rook Pawns without provocation.

\section{THE TOURNAMENT}

The tournament had 33 entrants, 3 over 2400 and 10 over 2300. Winning the tournament with 5 out of 6 games was Alexander Ivanov (2415 FIDE). Second place was shared by Mark Eidemiller, Klaus Pohl and Deep Thought with 4.5 (out of 6). For this performance, Deep Thought earned $\$ 567$ and a provisional rating of 2599 USCF. Deep Thought's wins were over players rated 2339, 2299, 2389 and 2491, losing only to Ivanov. Even more remarkable was the fact that in two of the games, Deep Thought suffered from hardware failures which caused it to move instantly. Fortunately the instant moves did not cause any serious problems. ChipTest scored 4 (out of 6), with a provisional rating of 2521 USCF, and picked up $\$ 100$. ChipTest was able to beat players rated 2353, 2417 and 2491. Hitech had a disappointing tournament, managing only 3.5 (out of 6) and a performance rating of 2325. This was Hitech's lowest performance rating in almost a year. BP scored 3 (out of 6), including a win and a draw against masters. BP's performance rating was 2185 .

Both Deep Thought and ChipTest played a number of "unusual" moves during the tournament, and it has been speculated that this caused their opponents to underestimate the programs. We will let the readers judge for themselves.

\section{REFERENCE}

Anantharaman, T., Campbell, M. and Hsu, F. (1988). Singular Extensions: Adding Selectivity to BruteForce Searching. Proceedings of the AAAI Spring Symposium, Computer Game Playing. AAAI, March, 1988. Also to appear in Artificial Intelligence.

\section{SOME GAMES}

\section{Deep Thought - Ross Sprague}

Sicilian Defense (B22)

1. e4 c5 2. c3 g6 3. d4 cxd4 4. cxd4 d5 5. e5 Nh6 6. Nc3 Nf5 7. Nf3 h5 8. Bd3 Nc6 9. Bf5 gxf5 10. Bf4 e6 11. O-O Bd7 12. a4 a6 13. Rc1 Qb6 14. Qd2 Na5 15. Qc2 Nb3 16. Rcd1 Rc8 17. Rd3 Qb4 18. Qb1 Rc4 19. Qd1 Na5 20. Qb1 Be7 21. Na2 Qb6 22. b3 Rc8 23. b4 Nc4 24. a5 Qb5 25. Rb3 Qc6 26. Nc1 Qc7 27. Nd3 Bb5 28. Qa2 Rg8 29. g3 h4 30. Ra1 Kd7 31. Rc1 hxg3 32. fxg3 Rg4 33. h3 Rg7 34. h4 Rh8 35. Qh2 Rh5 36. Kh1 Qd8 37. Qe2 R5h7 38. Nh2 Kc8 39. Rcc3 Kb8 40. Nf3 Rg4 41. Rb1 Qf8 42. Qh2 Qh8 43. Qc2 Rhg7 44. Qf2 Ka7 45. Nc5 Rh7 46 Nh2 Rg8 47. Qa2 Bxh4 48. gxh4 Rxh4 49. Qf2 Rgg4 50. Rf3 Na3 51. Rc1 Nc4 52. Rcc3 Qh5 53. Rcd3 Nb2 54. Rd2 Nc4 55. Ra2 Rg7 56. Nd3 R7g4 57. Bg3 Rxg3 58. Qxg3 Qh6 59. Qf2 Qh5 60. Ne1 Qg4 adj. 61. Ng2 (s) Rh7 62. Nf4 Qg8 63. Rg3 Qf8 64. Qe1 Rh4 65 Rf2 Qh6 66. Qc1 Qf8 67. Qc3 Qe7 68. Kg1 Qf8 69. Rg5 Qh6 70. Qg3 Ne3 71. Nh5 Re4 72. Nf6 Ng4 73. Nxe4 dxe4 74. Rxg4 Qc1+ 75. Nf1 fxg4 76. Qe3 Qd1 77. d5+ Black resigns. 
28. Thomas Martinak - Deep Thought

Slav Defense (D17)

1. d4 d5 2. c4 c6 3. Nf3 Nf6 4. Nc3 dxc4 5. a4 Bf5 6. Bg5 Qb6 7. Qd2 Nbd7 8. e3 Be6 9. e4 O-O-O 10. h3 Qb4 11. a5 Nc5 12. a6 bxa6 13. Qc2 Ncxe4 14. Bf4 Nd6 15. Rxa6 Qb7 16. Qa4 Nb5 17. Nxb5 cxb5 18. Qa5 Qe4+ 19. Be2 Qb1+ 20. Bd1 Qe4+ 21. Be2 Rd7 22. Bh2 Bd5 23. Rxa7 e5 24. Rxd7 Nxd7 25. dxe5 Qb1+ 26. Bd1 Qxb2 27. O-O Qb4 28. Qxb4 Bxb4 29. Nd4 Be4 30. Bg4 c3 31. Bf4 h5 32. Bxd7+ Kxd7 33. Nb5 c2 34. Ra1 Ra8 35. Rxa8 Bxa8 36. Bc1 Bc5 37. Kf1 Bb7 38. Nd6 Ba6+ 39. Kg1 Bxd6 40. exd6 Kxd6 41. f3 h4 42. Kf2 Kd5 43. g3 hxg3 44. Kxg3 Bd3 45. h4 g6 46. Kf4 f6 47. Ke3 Bf5 48. Bb2 Ke6 49. Kf4 Bh3 50. Ke4 Kd7 51. Kd3 Bf5+ 52. Ke3 Ke7 53. Ba3+ Ke6 54. Bb2 Kd7 55. Kd2 Ke7 56. Ke3 Bd7 57. Kd3 Ba4 58. Ke4 Kd6 59. Ba3+ Kc7 60. Bb2 Kd7 61. Kd3 Kd6 62. Ke3 Kd5 63. Kd2 f5 64. Ke3 Kc4 Draw.

42. Ronald Burnett - ChipTest

Scandinavian Defense (B01)

1. e4 d5 2. exd5 Qxd5 3. Nc3 Qa5 4. Nf3 Nf6 5. g3 Nc6 6. Bg2 e5 7. O-O Bb4 8. d3 Bxc3 9. bxc3 Qxc3 10. Rb1 O-O 11. Rb5 Qa1 12. Rb2 e4 13. Nd2 Bg4 14. Qe1 Nd4 15. Nb3 Nxb3 16. cxb3 Rad8 17. h3 Bf3 18. Bxf3 exf3 19. Qc3 Nd5 20. Qd4 c5 21. Qg4 Nb4 22. Qxf3 Nxd3 23. Rc2 b6 24. Bg5 Ne1 25. Qe2 Rd1 26. Kh1 Rb1 27. Rd2 h6 28. Be7 Re8 29. Qe4 Nf3 30. Qxb1 Qxb1 31. Rxb1 Nxd2 32. Rd1 Nxb3 33. Rd7 Nd4 34. Bd6 Re2 35. Rxa7 Rxf2 36. Kg1 Rd2 37. Bc7 b5 38. Bb6 Ne2+ 39. Kf2 c4 40. a3 Nc3+ 41. Ke1 Rh2 42. Ba5 Ne4 43. Bb4 Rxh3 44. g4 Re3+ 45. Kd1 Nf2+ 46. Kd2 Nxg4 47. Rb7 Rd3+ 48. Kc2 Ne3+ 49. Kb2 Rd5 50. Rc7 g5 51. Bc5 Rd2+ 52. Kc1 Rd1+ 53. Kb2 Nd5 54. Rb7 c3+ 55. Kc2 Rd2+ 56. Kc1 White resigns.

56. Hitech - Donald Meigs

French Defense (C10)

1. e4 e6 2. d4 d5 3. Nc3 dxe4 4. Nxe4 Nf6 5. Nxf6 gxf6 6. Nf3 Nc6 7. Be3 Bd7 8. d5 Ne5 9. dxe6 Bxe6 10. Be2 Bd6 11. Nd4 Qe7 12. Nxe6 fxe6 13. Qd4 Rg8 14. O-O-O Nc6 15. Qa4 Rxg2 16. Ba6! Rb8 17. Bxb7 Kf7 18. Bxc6 Rg6 19. Bxa7 Rb4 20. Qxb4 Bxb4 21. Rd7+ and White won.

72. ChipTest - Vivek Rao

Sicilian Defense (B22)

1. e4 c5 2. c3 d5 3. exd5 Qxd5 4. d4 Nf6 5. Nf3 Nc6 6. Be3 cxd4 7. cxd4 e6 8. Bd3 Be7 9. O-O Nb4 10. Nc3 Qd8 11. Bb5+ Bd7 12. Bxd7+ Qxd7 13. Ne5 Qd8 14. Qa4+ Kf8 15. Rc1 a6 16. Qb3 b6 17. Na4 b5 18. Nc5 Nbd5 19. Bd2 g6? 20. Bh6 Kg8 21. Nxe6! Black resigns (On 21. ... fxe6 22. Qh3 Qd6 23. Rc6).

73. Alexander Ivanov - Deep Thought

Scandinavian Defense (B01)

1. e4 d5 2. exd5 Qxd5 3. Nc3 Qa5 4. Nf3 Nf6 5. d4 c6 6. Bc4 Bg4 7. h3 Bh5 8. Qe2 Nbd7 9. Bd2 Qc7 10. O-O-O Bxf3 11. Qxf3 e6 12. g4 Bd6 13. g5 Nd5 14. Ne4 O-O 15. Nxd6 Qxd6 16. Qg3 Qxg3 17. fxg3 a5 18. Be2 b5 19. c4 bxc4 20. Bxc4 N7b6 21. Be2 Rfb8 22. Kc2 c5 23. dxc5 Na4 24. b3 Nxc5 25. Bf3 Rb5 26. Rc1 Rc8 27. Kb1 a4? 28. Bxd5 exd5 29. Be3 Rc6 30. Rxc5 Rbxc5 31. Bxc5 Rxc5 32. Rc1 Rxc1+ 33. Kxc1 a3 34. Kc2 Kf8 35. Kc3 Ke7 36. Kb4 Ke6 37. Kxa3 Ke5 38. Kb2 d4 39. Kc2 Ke4 40. Kd2 Black resigns.

74. Donald Meigs - BP

Three Knights (C46)

1. e4 e5 2. Nf3 Nf6 3. Nc3 Bb4 4. Bc4 O-O 5. d3 c6 6. O-O d5 7. Bb3 a5 8. a3 Bxc3 9. bxc3 a4 10. Ba2 Bg4 11. h3 dxe4 12. hxg4 exf3 13. gxf3 Nd5 14. Qe1 Nd7 15. Rb1 Qc7 16. Kg2 b6 17. Rh1 Qd6 18. c4 Ne7 19. Rb4 Rfe8 20. Be3 c5 21. Rb2 Ng6 22. Qd2 Re6 23. Bb1 h6 24. c3 Rf6 25. Bc2 Rxf3 26. Qd1 Nf4+ 27. Kf1 Qc6 28. Rg1 Ra7 29. Bxf4 Rxf4 30. g5 hxg5 31. Rxg5 Rxf2+ 32. Kxf2 Qf6+ 33. Qf3 Qxg5 
34. Qe3 Qxe3 35. Kxe3 g6 36. Bd1 f5 37. Bf3 f4+ 38. Ke4 Kf7 39. Kd5 Ke7 40. Kc6 Ra8 41. Rh2 Rc8+ 42. Kb5 Rd8 43. Kxa4 Nf6 44. Be2 f3 45. Bf1 g5 46. Kb5 Rd6 47. Rf2 g4 48. a4 e4 49. dxe4 Nxe4 50. Rc2 g3 51. a5 bxa5 52. Kxa5 Nd2 53. Bh3 g2 54. Rc1 Nb3+ White resigns.

\section{Deep Thought - Vivek Rao}

Sicilian Defense (B22)

1. e4 c5 2. c3 d5 3. exd5 Qxd5 4. d4 Nf6 5. Nf3 Nc6 6. Be2 cxd4 7. cxd4 e6 8. O-O Be7 9. Nc3 Qd8 10. Be3 O-O 11. Bc4 b6 12 a4 Bb7 13. Ne5 Rc8 14. Bb3 Nb4 15. Rc1 Nbd5 16. Nxd5 Bxd5 17. Qd3 Bb7 18. Rxc8 Qxc8 19. Rc1 Qa8 20. f3 Nd5 21. Bd2 Rc8 22. Bc4 Rc7 23. f4 Qc8 24. b3 f6 25. Ng4 Ba6 26. g3 Bb7 27. f5! a6 28. Rf1! Kh8 29. fxe6 Qxe6 30. Ne3 b5 31. axb5 axb5 32. Nxd5 Bxd5 33. Bxd5 Qxd5 34. Rf5 Qa8 35. Rxb5 Ra7 36. d5 Ra1+ 37. Kg2 Ra2 38. b4 Qc8 39. Ra5 Rb2? 40. Qc3 Black resigns.

\section{Deep Thought - Romuel Reyes}

French Defense (C01)

1. e4 e6 2. d4 d5 3. Nc3 Bb4 4. exd5 exd5 5. Bd3 Ne7 6. Ne2 c6 7. O-O O-O 8. Re1 Bg4 9. f3 Bc8 10. Bg5 f6 11. Bf4 Re8 12. Ng3 Nd7 13. a4 Nf8 14. Be3 Nfg6 15. f4 Bd6 16. Qh5 Bd7 17. b3 Qc8 18. h3 Qc7 19. Nce2 Rad8 20. Rf1 Nc8 21. Rf3 Nf8 22. c4 dxc4 23. bxc4 g6 24. Qh6 Be6 25. f5 Bf7 26. Ne4 Rxe4 27. Bxe4 Bxc4 28. Nc3 Qg7 29. Rb1 Rd7 30. Qh4 g5 31. Qe1 Ne7 32. Qd2 h6 33. Bd3 Bf7 34. Ne4 Nc8 35. h4 Bd5 36. hxg5 hxg5 37. Rh3 Re7 38. Nxd6 Nxd6 39. Qb4 Rd7 40. Re1 Qf7 41. a5 Bc4 42. Bc2 Nb5 43. Qc5 Qd5 44. Qxd5 Bxd5 45. Bd3 a6 46. Rh6 Kg7 47. Rh2 Bf7 48. Bxb5 axb5 49. Rb1 Re7 50. Bd2 Re4 51. Bc3 Nd7 52. Re1 Rxe1 53. Bxe1 Nb8 54. Rh3 Na6 55. Re3 Kf8 56. Bg3 c5 57. Bd6+ Kg8 58. dxc5 b4 59. Be7 Kg7 60. Rd3 b3 61. Rd7 Nb4 62. Rxb7 Nc6 63. a6 Bd5 64. Bd8+ Black resigns.

\section{ChipTest - Klaus Pohl}

French Defense (C01)

1. e4 e6 2. d4 d5 3. Nc3 Bb4 4. exd5 exd5 5. Bd3 Nc6 6. Nge2 Bg4 7. f3 Bh5 8. O-O Bg6 9. Re1 Nge7 10. a3 Ba5 11. Bxg6 hxg6 12. b4 Bb6 13. b5 Na5 14. Bg5 f6 15. Nf4 Kf7 16. Ne6 Qd7 17. Bf4 Rae8 18. Qd3 Nc4 19. Nc5 Qf5 20. Ne2 Qxd3 21. cxd3 Nd6 22. Bxd6 cxd6 23. Nxb7 Nf5 24. g4 Rxe2 25. Rxe2 Bxd4+ 26. Kg2 Nh4+ 27. Kg3 Bxal 28. Nxd6+ Kg8 29. Re8+ Kh7 30. Rxh8+ Kxh8 31. Kxh4 Kg8 32. Nc8 Bd4 33. Ne7+ Kf7 34. Nxd5 g5+ 35. Kg3 Ke6 36. Nb4 Bc5 37. Nc2 Kd5 38. Kg2 Bd6 39. h3 Kc5 40. a4 Kb6 41. Ne3 Be5 42. Nc4+ Kc5 43. Nxe5 fxe5 44. Kf2 Kb4 45. Ke3 Kxa4 46. Kd2 Kxb5 47. Kc3 Kc5 48. Kb3 $\mathrm{Kd} 449 . \mathrm{Kc} 2$ a5 50. Kd2 a4 White resigns.

Fredkin Masters Open

Pittsburgh, Pennsylvania

May 28-30, 1988

$\begin{array}{rlrrrrrrrr} & \text { Rating } & 1 & 2 & 3 & 4 & 5 & 6 & \text { Pts } \\ 1 & \text { Rao, Vivek } & 2491 & 16 \mathrm{w}= & 27 \mathrm{~b}+ & 9 \mathrm{~b}+ & 3 \mathrm{w}= & 20 \mathrm{~b}- & 21 \mathrm{~b}- & 3 \\ 2 & \text { Burnett, Rbnald } & 2417 & * & 16 \mathrm{~b}+ & 20 \mathrm{w}- & 23 \mathrm{w}+ & 10 \mathrm{~b}- & --- & 2.5 \\ 3 & \text { Ivanov, Alexander } & 2415 & 17 \mathrm{~b}+ & 15 \mathrm{w}+ & 4 \mathrm{~b}+ & 1 \mathrm{~b}= & 21 \mathrm{w}+ & 7 \mathrm{w}= & 5 \\ 4 & \text { Reyes, Roumel } & 2389 & 18 \mathrm{w}+ & 14 \mathrm{~b}+ & 3 \mathrm{w}- & 21 \mathrm{~b}- & 12 \mathrm{w}= & 17 \mathrm{w}= & 3 \\ 5 & \text { Hitech, Compx } & 2376 & 19 \mathrm{~b}- & 18 \mathrm{w}+ & 15 \mathrm{~b}= & 16 \mathrm{w}+ & 6 \mathrm{w}= & 12 \mathrm{~b}= & 3.5 \\ 6 & \text { Leverett, Bruce } & 2353 & 20 \mathrm{w}- & 22 \mathrm{~b}+ & 19 \mathrm{w}+ & 12 \mathrm{w}= & 5 \mathrm{~b}= & 14 \mathrm{w}+ & 4 \\ 7 & \text { Eidemiller, Mark } & 2352 & * & 30 \mathrm{w}+ & 13 \mathrm{~b}+ & 20 \mathrm{w}= & 14 \mathrm{~b}+ & 3 \mathrm{~b}= & 4.5 \\ 8 & \text { Sprague, Ross } & 2339 & 21 \mathrm{~b}- & 24 \mathrm{w}- & 28 \mathrm{~b}+ & 19 \mathrm{w}+ & 17 \mathrm{w}+ & 13 \mathrm{w}- & 3 \\ 9 & \text { Rolletschek, Heinrich } & 2334 & 22 \mathrm{w}+ & 19 \mathrm{~b}+ & 1 \mathrm{w}- & 14 \mathrm{~b}- & 23 \mathrm{~b}+ & 27 \mathrm{w}- & 3 \\ 10 & \text { Pohl, Klaus } & 2321 & 23 \mathrm{~b}- & 25 \mathrm{w}+ & 17 \mathrm{~b}= & 15 \mathrm{w}+ & 2 \mathrm{w}+ & 20 \mathrm{~b}+ & 4.5 \\ 11 & \text { Nedved, Kimball } & 2299 & 24 \mathrm{w}+ & 20 \mathrm{~b}= & 21 \mathrm{w}- & 17 \mathrm{w}= & --- & --- & 2 \\ 12 & \text { Martinak, Tom } & 2292 & 25 \mathrm{~b}+ & 21 \mathrm{w}= & 23 \mathrm{w}= & 6 \mathrm{~b}= & 4 \mathrm{~b}= & 5 \mathrm{w}= & 3.5 \\ 13 & \text { Wheeler, Jerry } & 2281 & 26 \mathrm{w}+ & 23 \mathrm{~b}= & 7 \mathrm{w}- & 27 \mathrm{~b}= & 18 \mathrm{w}+ & 8 \mathrm{~b}+ & 4 \\ 14 & \text { Kurzdorfer, Peter } & 2243 & 28 \mathrm{~b}+ & 4 \mathrm{w}- & 24 \mathrm{~b}+ & 9 \mathrm{w}+ & 7 \mathrm{w}- & 6 \mathrm{~b}- & 3\end{array}$




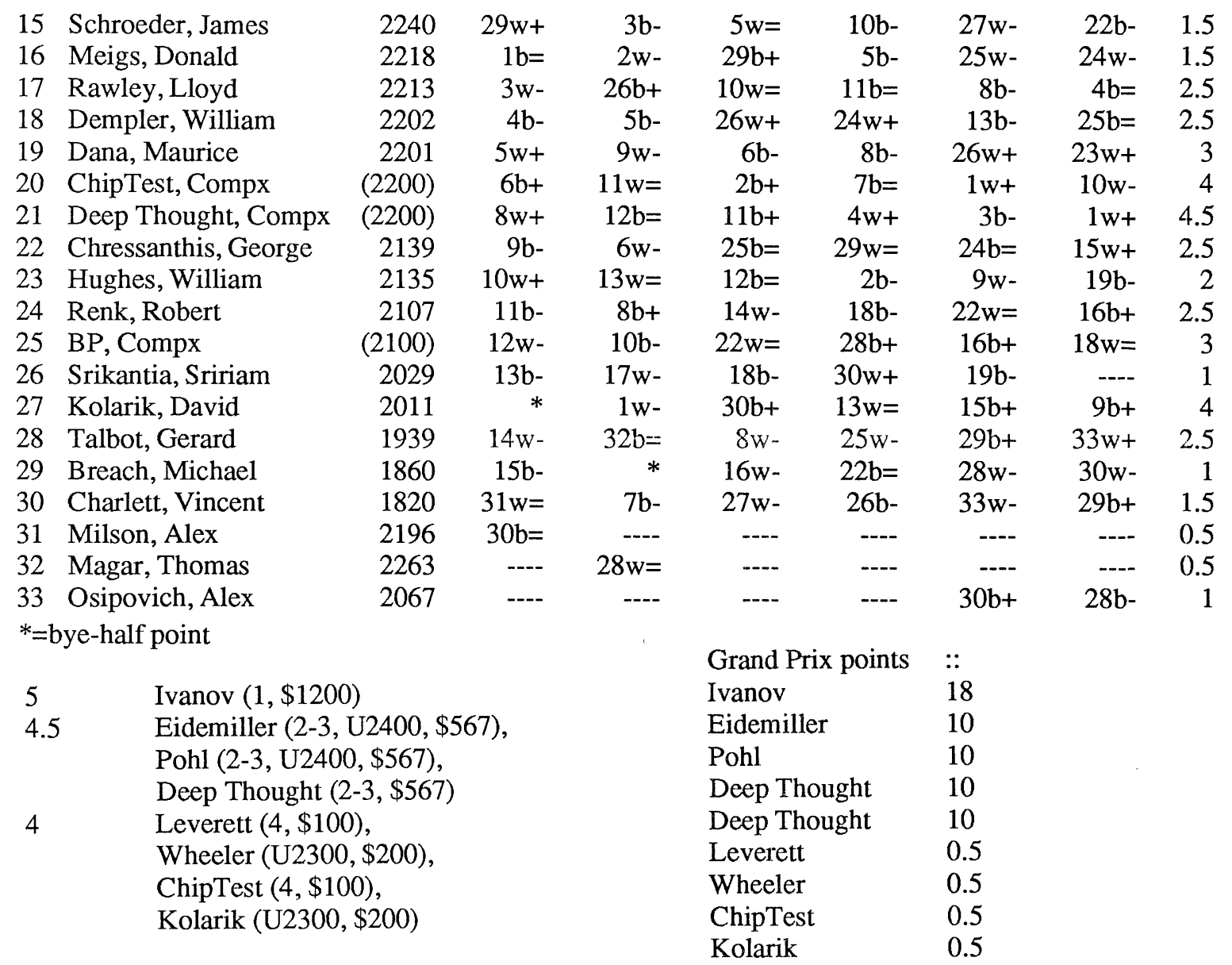

Performance, Old and New Ratings based on last published ratings

$\begin{array}{lrrrr}\text { Ivanov } & 5 & 2643 & 2415 / 1 & 2610 / 7 \\ \text { Deep Thought } & 4.5 & 2571 & 0 & 2571 / 6 \\ \text { ChipTest } & 4 & 2506 & 0 & 2506 / 6 \\ \text { Eidemiller } & 4.5 & 2493 & 2352 & 2378 \\ \text { Pohl } & 4.5 & 2483 & 2321 & 2349 \\ \text { Leverett } & 4 & 2426 & 2353 & 2365 \\ \text { Martinak } & 3.5 & 2402 & 2292 & 2312 \\ \text { Kolarik } & 4 & 2393 & 2011 & 2080 \\ \text { Nedved } & 2 & 2349 & 2299 & 2304 \\ \text { Rao } & 3 & 2343 & 2491 & 2476 \\ \text { Reyes } & 3 & 2323 & 2389 & 2376 \\ \text { Hitech } & 3.5 & 2318 & 2376 & 2364 \\ \text { Wheeler } & 4 & 2311 & 2281 & 2287 \\ \text { Burnett } & 2.5 & 2295 & 2417 & 2407 \\ \text { Dana } & 3 & 2261 & 2201 & 2213 \\ \text { Kurzdorfer } & 3 & 2246 & 2243 & 2245 \\ \text { Rawley } & 2.5 & 2232 & 2213 & 2218 \\ \text { Sprague } & 3 & 2219 & 2339 & 2318 \\ \text { Rolletschek } & 3 & 2203 & 2334 & 2311 \\ \text { BP } & 2.5 & 2185 & 0 & 2185 / 6 \\ \text { Hughes } & 2 & 2174 & 2135 & 2143 \\ \text { Renk } & 2.5 & 2173 & 2107 & 2121 \\ \text { Dempler } & 2.5 & 2161 & 2202 & 2195 \\ \text { Chressanthis } & 2.5 & 2113 & 2139 & 2136 \\ \text { Talbot } & 2.5 & 2093 & 1939 & 1971 \\ \text { Meigs } & 1.5 & 2039 & 2218 & 2187 \\ \text { Schroeder } & 1.5 & 1987 & 2240 & 2197 \\ \text { Magar } & 0.5 & 1939 & 2263 & 2254\end{array}$




$\begin{array}{lrrrr}\text { Srikantia } & 1 & 1903 & 2029 & 2005 \\ \text { Osipovich } & 1 & 1880 & 2067 & 2052 \\ \text { Milson } & 0.5 & 1820 & 2196 & 2186 \\ \text { Charlett } & 1.5 & 1753 & 1820 & 1796 \\ \text { Breach } & 1 & 1751 & 1860 & 1834\end{array}$

$\begin{array}{llll}\text { FIDE ratings } & & & \\ \text { Ivanov, Alexander } & 2420 & & \text { URS } \\ \text { Leverett, Bruce } & 2245 & & \text { USA } \\ \text { Rao, Vivek } & 2355 & \text { FM } & \text { USA } \\ \text { Reyes, Romuel } & 2320 & & \text { PHI }\end{array}$

Actual ratings at tourney time:

$\begin{array}{ll}\text { HITECH-2381 } \\ \text { Dana } & 2211 \\ \text { Dempler } & 2218 \\ \text { Schroeder } & 2232 \\ \text { Meigs } & 2212 \\ \text { Leverett } & 2380 \\ \text { Martinak } & 2297\end{array}$

$\begin{array}{lr}\text { CHIPTEST-2521 } \\ \text { Leverett } & 2380 \\ \text { Nedved } & 2307 \\ \text { Burnett } & 2431 \\ \text { Eidemiller } & 2353 \\ \text { Rao } & 2491 \\ \text { Pohl } & 2364\end{array}$

DEEPTHOUGHT-2599

Sprague 2324

Martinak 2297

Nedved 2307

Reyes $\quad 2378$

Ivanov 2597

Rao $\quad 2491$

\title{
THE $1^{\text {st }}$ COMPUTER OLYMPIAD
}

\author{
Park Lane Hotel, London \\ August 9-15, 1989 \\ ICCA Communication
}

by David Levy

Further to the first announcement in the ICCA Journal (Vol. 10, No. 2, p. 105) on the Computer Olympiad some more details can be provided.

The world's first Olympiad for computer programs will take place at the Park Lane Hotel, London, from August 9th to 15th 1989. This unique event will feature tournaments for chess, bridge, backgammon, draughts, poker, Go, and many other classic "thinking" games. In every tournament all of the competitors will be computer programs. The role of the human operators will merely be to tell their own programs what moves have been made by their opponents. The Computer Olympiad is organized by International Chess Master David Levy, who is President of the International Computer Chess Association. Anyone wanting more information on the event should send a large, stamped addressed envelope to: Computer Olympiad, 11 Loudoun Road, London NW8 0LP.

\section{Call for Papers}

The 1st London Conference on Computer Games will take place as part of the Computer Olympiad during the period August 9th to 15th, 1989. Papers are invited on any aspect of programming computers to play "thinking" games such as chess, bridge, Go, backgammon, etc. The conference Chairman will be Professor Tony Marsland, from the Computing Science Department at the University of Alberta, Edmonton, Canada. The editor of the conference proceedings will be Don Beal, of the Computer Science Department at Queen Mary College, London University. Papers should preferably be 3,000 to 4,000 words in length, and if possible should be submitted together with an IBM PC format disk containing the text as a Wordstar file. The closing date for submission is May 9th 1989. Papers should be sent to: Computer Olympiad, 11 Loudoun Road, London NW8 0LP, England. 